Straight imaging and mechanism behind grain boundary electron emission in Pt-doped ultrananocrystalline diamond films Peer-reviewed author version

Panda, Kalpataru; Inami, Eiichi; Sugimoto, Yoshiaki; KAMATCHI JOTHIRAMALINGAM, Sankaran \& Lin, I-Nan (2017) Straight imaging and mechanism behind grain boundary electron emission in Pt-doped ultrananocrystalline diamond films. In: CARBON, 111, p. 8-17.

DOI: 10.1016/j.carbon.2016.09.062

Handle: http://hdl.handle.net/1942/23232 


\title{
Straight Imaging and Mechanism behind Grain Boundary Electron Emission in Pt-doped Ultrananocrystalline Diamond Films
}

Kalpataru Panda ${ }^{1, \dagger}$, Eiichi Inami ${ }^{2}$, Yoshiaki Sugimoto ${ }^{1}$, K. J Sankaran ${ }^{3}$, I-Nan Lin ${ }^{4}{ }^{\dagger}$

${ }^{1}$ Department of Advanced Materials Science, University of Tokyo, Kashiwa, Chiba, 277-8561, Japan;

${ }^{2}$ Research Center for Micro-Nano Technology, Hosei University, Tokyo 184-0003, Japan

${ }^{3}$ Institute for Materials Research (IMO), Hasselt University, 3590, Diepenbeek, Belgium

${ }^{4}$ Department of Physics, Tamkang University, Tamsui 251, Taiwan, ROC

${ }^{\dagger}$ Contact Author: phy.kalpa@gmail.com, inanlin@mail.tku.edu.tw

\begin{abstract}
A detailed scanning tunneling microscopic (STM) investigation is carried out to directly image and understand the mechanism behind enhanced conductivity and electron field emission (EFE) properties for platinum (Pt) ion doped/post-annealed ultrananocrystalline diamond (UNCD) films. Straight imaging of conducting/nonconducting sites is mapped by dynamic scanning tunneling microscopy (D-STM). Energy dissipation mapping and the local current-voltage measurements illustrate that grain boundaries are the conducting/emitting sites. Further, this fact is supported by the high resolution current imaging tunneling spectroscopy (CITS). The formation of abundant conducting $\mathrm{sp}^{2}$ nanographitic phases along the diamond grain boundaries, confirmed from transmission electron microscopic examinations, is believed to be the genuine factor that helps for the easy transport of electrons and hence enhanced the conductivity/emission properties. The fabrication of these films with high conductivity and superior EFE properties is a direct and simple approach which opens
\end{abstract}


up new prospects in flat panel displays and high brightness electron sources. Moreover, we expect the energy dissipation associated with assisted tunneling process of electrons from the tip of STM to sample surface, can be a very useful technique for imaging heterostructured semiconducting surfaces due to their electrical conductivity differences in nanometer scale, which can explain many physical and chemical properties.

\section{Introduction}

Cold cathode materials with excellent electron field emission (EFE) properties have great promise for use in vacuum electronic devices such as electron sources for electron microscopes, $\mathrm{X}$-ray sources, high energy accelerators, cathode-ray tube monitors and microwave amplifiers [1-3]. Diamond is a good electronic candidate for solid-state electronic emitters because of its negative electron affinity (NEA) and low effective work function surface [4,5]. Ultrananocrystalline diamond (UNCD) film, which has ultra-small diamond grain sizes of 2-5 $\mathrm{nm}$ with smooth surfaces, has recently attracted broad interest because of its excellent EFE properties as compared to microcrystalline and nanocrystalline diamond films [6,7]. The diamond or UNCD films have to be conductive, for the purpose of using it as a material for the fabrication of cold cathode/electron emitting devices [8-10]. Many groups have followed the doping method to make conducting UNCD films with better EFE properties [8,9]. The doping of nitrogen $\left(\mathrm{N}_{2}\right)$ during the growth plasma can effectively improve the electrical conductivity and EFE properties of UNCD films [8-10]. It is believed that the conversion of an amorphous carbon (a-C) phase to a conducting graphitic phase along the diamond grain boundaries enhance the efficiency for transporting electrons on the surface and hence improved the conductivity/EFE properties for UNCD films $[8,10,11]$. The grain boundary conduction mechanism is believed to be responsible for the enhanced EFE properties for $\mathrm{N}_{2}$ doped UNCD films [11-13].

Many groups proposed the possibilities of conduction channel and/or grain-boundary conduction mechanism for the enhanced conductivity/electron emission properties for doped polycrystalline diamond thin films [14-16]. The conductivity/electron emission properties is believed to correlate with the number density of grain boundaries containing non-diamond $s p^{2}$ 
phases [17]. To clarify this, Cui et al. [18] proposed a mechanism that the threshold for electron field emission is lowered due to a local reduction of the electron affinity at the nanostructured diamond surface surrounded by the nanoscale grain boundaries containing graphitic phases. They reported that electron emission takes place from the graphitic grain boundaries, but the emission barrier is controlled by the surrounding diamond surface. Robertson [19] and Ilie [20] suggested similar model for the enhanced field emission from other forms of carbon samples, including flat, smooth diamond-like carbon (DLC).

Few attempts have been tried by different groups to find out the exact location of electron emission sites on diamond surfaces. Grain boundary electron emission was explained by May and co-workers [21] by using intentional high input bias to burn out the emission sites in diamond films. Ring-like craters observed in the damaged emission sites surrounding the diamond grains $(\mathrm{G})$, showed that the grain boundaries are the electron emission sites, damaged due to the passage of excess current. Further, by using field electron emission microscopy (FEEM), the field-emitted electrons are collected and imaged, enabling their origin to be determined. By using FEEM, Garguilo found that the electron emission sites were originated from localized regions smaller than $100 \mathrm{~nm}$ in size for microcrystalline diamond (MCD) films [22]. Unfortunately, FEEM has proved to be tough to implement on UNCD surfaces, because of its insufficient resolution to accurately determine the exact location of the emission sites. On the other hand, the electron field emission and surface electronic properties of doped DLC and nanostructured diamond films were addressed by utilizing the scanning tunneling microscopy (STM) [23-26]. These studies report the surface electronic properties of doped UNCD in a local scale. Only few studies have reported on the relationship between the microstructure and electron emission properties [25, 26]. However, there is no experimental studies which report the relationship between the direct observations of the electron emission sites with its microstructures for doped UNCD. In this context, a clear understanding of the relationship between the conducting/electron emitting sites with its microstructure is required to reveal the mechanism behind enhanced conductivity/EFE properties for doped UNCD films.

Up to date, few studies report the direct observation of emission sites by using STM to simultaneously map the surface topography and the emission sites on diamond surfaces. Using STM, Krauss and co-workers [27] reported that the emission sites from UNCD coated Si tips, are 
related to the inflection points or grain boundaries in UNCD surface, and not to surface asperities or grains. However, the electron emission map from a $6 \times 6 \mu \mathrm{m}^{2}$ sample surface makes it difficult to detect the actual emission sites. By using STM, Karabutov et al. [28] observed that the emission sites from microcrystalline diamond films were not due to the sharp morphology protrusions (grains) but instead the morphology pits (grain boundaries). However, the data from only one sample with $400 \times 400 \mathrm{~nm}$ scale, it is hard to point out whether the diamond grains or grain boundaries were the actual conducting/emitting sites. Recently Harniman et al., [29] and V. Chatterjee et al. [30] showed that the grain boundaries are the prominent electron emission sites for polycrystalline diamond films by using PeakForce-controlled tunneling atomic force microscopy (PF-TUNA) in ambient conditions, where the topographic and tunneling current information were collected under PeakForce feedback. However, in ambient conditions, the surface deformation mediated by surface contaminations and surface conducting layers, drastically affect the tunneling current and hence for the real detection of the electron emission sites [29, 31]. Moreover, from the PF-TUNA experiments in ambient conditions, it is very hard to find out whether the nanometer sized diamond grain boundaries were the real emission sites $[29,30]$. Therefore, it is important to carry out the measurements in ultra-high vacuum (UHV) conditions to avoid the effect of surface contaminants and surface conducting layers present in ambient conditions on the tunneling current and hence to detect the real conducting/emitting sites.

In this context, we utilize a new STM technique, energy dissipation measurement in dynamic STM (D-STM) mode, in UHV conditions to directly image the conducting/nonconducting sites for Pt ion doped/post-annealed UNCD films in nanometer scale. Moreover, current imaging tunneling spectroscopy (CITS) measurements are carried out in UHV conditions to identify the prominent electron emission sites. Since both the energy dissipation and CITS measurements are measured in UHV condition, the accurate detection of the tunneling current and hence the exact location of the conducting/emitting sites is possible in high resolution with much accuracy. Moreover, the effect of microstructure on the enhanced conductivity/emission properties are discussed by TEM. 


\section{Experimental}

The UNCD films with ultra-smooth surface characteristics were grown on n-type silicon substrates by a microwave plasma enhanced chemical vapor deposition system $(2.45 \mathrm{GHz}$ IPLAS-CYRANNUS-I, Troisdorf, Germany), using $\operatorname{Ar}(99 \%) / \mathrm{CH}_{4}(1 \%)$ gas at $1200 \mathrm{~W}$ and 120 Torr. A solution containing a mixture of diamond powders and titanium powders (SIGMAALDRICH; 365 mesh) were used for the ultrasonication of the Si substrates for 45 minutes to facilitate the nucleation process before the growth of diamond films on it. Platinum ions were implanted on UNCD films at room temperature with the implantation energy of $500 \mathrm{keV}$ and ion fluence of $1 \times 10^{17}$ ions $/ \mathrm{cm}^{2}$ at a base pressure below $2 \times 10^{-7} \mathrm{mbar}$, followed by annealing at $800^{\circ} \mathrm{C}$ for 30 minutes. For simplicity, the pristine UNCD films are designated as "as-UNCD," whereas the Pt ion doped/post-annealed films as "doped-UNCD". The EFE experiments were carried out using a homemade tunable parallel plate capacitor setup, where a molybdenum rod of $2 \mathrm{~mm}$ diameter was used as a anode. The cathode (UNCD films) to anode (Molybdenum rod) distance is controlled by a micrometer and an optical microscope. Hall measurements were carried out in a van der Pauw configuration (ECOPIA HMS-3000) to observe the conducting behavior of these films. Energy dissipation measurements were performed by a custom-built combined non-contact atomic force microscope (NC-AFM) and dynamic scanning tunneling microscopic (D-STM) system in ultrahigh-vacuum $\left(5 \times 10^{-11}\right.$ torr $)$ conditions operated at RT, to detect the conducting/non-conducting sites. Dissipation signal was taken while oscillating the cantilever at constant amplitude. Conductive Pt coated Si cantilevers were cleaned via $\mathrm{Ar}^{+}$ion sputtering to remove the native oxide layer and other contaminants prior to D-STM imaging and characterizations. CITS mapping with voltages ramping from -3.3 to $3.3 \mathrm{~V}$ were acquired concurrently during the STM topography scanning at a fixed height of tip over the examined region. Transmission electron microscopy (TEM; JEOL 2100F) was used to examine the microstructure of the as-UNCD and doped-UNCD films. 


\section{Results and Discussions}

\subsection{Results}

The enhancement in electron field emission and conductivity properties of as-UNCD films after the Pt ion doping/post-annealing processes are summarized in Table 1. A significant enhancement in EFE properties is observed for as-UNCD films after the Pt ion doping/postannealing processes. The turn-on field $\left(\mathrm{E}_{0}\right)$, defined as the lowest electric field at which the electrons start to emit from the sample surface, is assigned as the interception of the straight lines extrapolated from the low field and high field segments of the $\mathrm{F}-\mathrm{N}$ plots. The $\mathrm{E}_{0}$ value decreased from $22.2 \mathrm{~V} / \mu \mathrm{m}$ in case of as-UNCD to $4.17 \mathrm{~V} / \mu \mathrm{m}$ for doped-UNCD films. Such kind of EFE properties of doped-UNCD films are comparable to that of conducting UNCD films ever reported (see Table I) [11, 32-35]. Moreover, the I-V curves by 4-probe technique shows that the as-UNCD films are too resistive (curve I, Fig. S1, Supplementary information), while the dopedUNCD films (curve II, Fig. S1, Supplementary information) have metallic conductivity. Further, Hall measurements confirm, doped-UNCD films have a high electrical conductivity of 95.0 $\mathrm{ohm}^{-1} \mathrm{~cm}^{-1}$, while as-UNCD films are resistive enough to be measured by this technique, as shown in Table I.

In NC-AFM/dynamic scanning tunneling microscopy (D-STM), the oscillation energy of a cantilever can be dissipated depending on the electrical nature of the sample surface when the tip approaches the sample surface $[36,37]$. The resistance offered to the tunneling current by the resistive nature of sample surface is one of the main factors of this dissipation in a local scale. Previous studies suggest that the electrical dissipation is related to the displacement current induced by the cantilever motion [36, 38]. Denk and Pohl attributed the dissipation variations in imaging heterostructured semiconducting surfaces to the Joule dissipation which arises due to the local resistance on the sample surface [36]. By using the dissipation variations, they observed strong damping contrast in layered GaAs/AlGaAs semiconductor heterostructures due to the difference in conducting properties of GaAs and AlGaAs. They concluded that the dissipation depend on the type of material, dopant concentration, illumination, and the applied voltage to sample during scanning. Fukuma and co-workers reported that the electrical dissipation strongly depend on the resistance of the bias line during the scanning, revealing that the displacement current in the bias line plays an important role in the electrical dissipation process [39]. By 
varying the resistance of the bias line during imaging oligothiophene sub-monolayer films deposited on a Pt substrate, they noticed strong contrast on the dissipation images. Stowe et al. quantitatively analyzed the electrical dissipation of a laterally vibrated probe as a function of dopant density, [38] which supported the Joule dissipation model. They showed that some local charge flow induced by the lateral vibration of the cantilever lead to the Joule dissipation at the vicinity of the sample surface. Moreover, the energy dissipation method is found to be very suitable to directly map the conducting/emitting sites for doped-UNCD surfaces locally. The variation in energy dissipation is expected during scanning a doped-UNCD, because the displacement current in the bias line seems to be different as the sample surface contains conducting graphitic, non-conducting diamond, a-C and hydrocarbon phases.

Fig. 1(a) shows the D-STM topography image of doped-UNCD surface with the corresponding dissipation image in Fig. 1(b). The black and yellowish color in Fig. 1(b) represent the lower and higher dissipation channels on the surface. It is observed that the black river-like channel marked as "GB1" surrounds the diamond grain marked as "G1" in Fig. 1(b), indicating that the grain boundaries (GB1) are the lower dissipative channels comparing to the diamond grains (G1). Moreover, few nanoclusters, marked as " 2 " in the topography in Fig. 1(a), is seen as black in the dissipation image in Fig. 1(b). These nanoclusters are presumed to be metallic Pt nanoclusters and hence lower dissipative in nature. The appearance of Pt nanoclusters on the surface of doped-UNCD films is resulted from the post-annealing of the Pt ion doped UNCD films, which is discussed by TEM shortly. Moreover, energy dispersive x-ray analysis (EDX) clearly shows the presence of Pt nanoclusters on doped-UNCD surface (Fig. S2, Supplementary information). A dissipation energy difference of $\sim 2 \mathrm{eV}$ is measured between $\mathrm{G} 1$ and GB1. The reason for the difference in dissipation energy is apparently due to the different resistive nature at the grain and grain boundaries. This is known as Joule dissipation which normally arises when the sample surface consists of conducting and less conducting channels on the surface $[36,38,39]$. Further, to confirm whether the diamond grain or grain boundaries are the conducting channels, local $\mathrm{I}-\mathrm{V}$ characteristic curves were recorded at these positions during the high resolution STM topography scanning, as shown in Fig. 1(c). Tunneling current is observed to be $0.5 \mathrm{nA}$ at the GB at a sample bias of $-3.5 \mathrm{~V}$, whereas it is almost zero at the $\mathrm{G}$ at 
the same bias. Therefore, the dissipation image in Fig. 1(b) and the reproducible I-V curves in Fig. 1(d) depicts that the grain boundaries are the prominent conducting channels.

Fig. 2 explains the dissipation mechanism along with the results schematically. Fig. 2(a) shows the schematic of the dissipation mechanism. A vibrating cantilever in D-STM mode image the sample surface as the tunneling electrons flow from the sample surface to the tip. The dissipation energy is observed to be higher at the grain (marked as "G1" in Fig. 1(b)) comparing to the grain boundaries (marked as "GB1" in Fig. 1(b)), shown schematically in Fig. 2(b). It seems that the tunneling electrons at the non-conducting grains cannot transport as easily as at the conducting grain boundaries and hence dissipates more energy as heat at the grains. Since the resistance offered to the tunneling electrons at the grains $\left(\mathrm{R}_{\mathrm{G}}\right)$ is more than the grain boundaries $\left(\mathrm{R}_{\mathrm{GB}}\right)$, hence the dissipation energy is more for the grains than the grain boundaries. Restated, tunneling electrons is assumed to pass easily through the grain boundaries while they find resistance at the grains.

To reveal the effect of doping/post-annealing processes on the conductivity and EFE properties, the local electronic properties of doped-UNCD films were investigated by CITS in STS mode. It should be noted that the surface of as-UNCD films are too resistive for STM measurements. So the surface is sputtered etched insitu in UHV conditions for $1 \mathrm{~min}$., so as to render the surface conductive for STM measurements. The STM topography of as-UNCD and doped-UNCD are shown in Figs. 3(a) and 3(c), respectively, while the corresponding CITS images, are shown in Figs. 3(b) and 3(d), taken at a sample bias of $-3 \mathrm{~V}$. The shape of the bright and dark regions in the CITS images in Figs. 3(b) and 3(d) have similarity with the shapes of the STM topography in Figs. 3(a) and 3(c) respectively. Bright regions in CITS image reveal better electron emission sites [40]. The typical grain and grain boundaries are marked as "G1", "G2" and "GB1", "GB2", respectively, in the STM image in Fig. 3(a) with their corresponding marking in the CITS image in Fig. 3(b) for as-UNCD films. Similarly, the grain and grain boundaries are marked as "G3", "G4" and "GB3", "GB4", respectively, in Fig. 3(c) with their corresponding marking in Fig. 3(d) for doped-UNCD films. Interestingly, the electron emission sites are observed to be from the grain boundaries of diamond aggregates, GB1, GB2 in asUNCD as marked in Fig. 3(b) and GB3, GB4 in doped-UNCD, in Fig. 3(d). Moreover, it is very 
clear that the number density of emission sites significantly enhanced in doped-UNCD in Fig. 3(d) than as-UNCD (cf. Fig. 3(b)), such that it looks as if the whole surface is emitting, which implies that the surface of doped-UNCD films become more conducting.

The high resolution STM (HRSTM) image of doped-UNCD film along with the corresponding CITS mapping were recorded to reveal the exact locations of emission sites in a local scale. Fig. 4(a) shows the HRSTM image with the corresponding CITS mapping in Fig. 4(b), taken at a sample bias of $-3 \mathrm{~V}$. Bright regions are observed along the grain boundaries marked as "GB5" and "GB6" in Fig. 4(b), indicating that the grain boundaries are the prominent emitting/conducting sites. The corresponding grains marked as "G5" and "G6" look dark in the CITS image in Fig. 4(b), indicating lower emission or less conducting than those of the grain boundaries. Moreover few nanoclusters marked as "7" in Fig. 4(a) look bright in the corresponding CITS image in Fig. 4(b), indicating that they also emit like the grain boundaries. The appearance of these high electron emitting clusters might be the platinum nanoclusters which appear on the surface of doped-UNCD films due to the post-annealing of the Pt ion doped UNCD films. Interestingly, these Pt nanoclusters look dark in the dissipation image in Fig. 1(b), indicating that they are less dissipative and conducting in nature, which well supports the CITS results in Fig. 4(b). Further, the three-dimensional (3D) surface topography representation of doped-UNCD in Fig. 4(a) and the corresponding CITS image in Fig. 4(b), are shown in Fig. 4(c) and Fig. 4(d), respectively. The 3D surface topography in Fig. 4(c) and the corresponding CITS image in Fig. 4(d) are rotated by $45^{\circ}$ for better representation. The corresponding grain and grain boundaries in the 3D images are marked both in the topography and the corresponding CITS image. The dark and bright contrast in Fig. 4(d) represents the lower and higher electron emission and corresponds to the grain and grain boundaries in Fig. 4(c), respectively. It clearly indicates that the grain boundaries are the prominent electron emitting/conducting sites. Further, local current-voltage (I-V) characteristic curves were taken at the grain and grain boundaries for doped-UNCD films to crosscheck the emission properties observed by CITS. The I-V curves were taken when the tip is held constant over the surface i.e., static STM (S-STM), while the curves shown in Fig. 1(d) were taken by a dynamic cantilever tip i.e. D-STM mode. Local I-V curves were recorded during the HRSTM image scanning at the grain and grain boundaries, as shown in Fig. 4(e). Only the negative portion of the I-V curves, which indicate the emission of 
electrons from the film surface, is shown here. Ten reproducible I-V curves corresponding to each emission sites as G (curve I), Pt nanoparticles (curve II) and GBs (curve III) of dopedUNCD films is shown in Fig. 4(e). The open symbols in the I-V curves are the reproducible curves measured in different STS measurements and the solid curves are the average of these reproducible curves. Interestingly, a significant change in I-V characteristic curves at the grain (G) and grain boundaries (GBs) is observed. The GBs (curve III, Fig. 4(e)) are always observed to emit at a lower sample bias compared to the grains (curve I, Fig. 4(e)). Moreover, few Pt nanoclusters, marked as "7" in Fig. 4(a) are also seen to emit at a lower sample bias (curve II, Fig. 4(e)), in addition to the grain boundaries. The local I-V results from both S-STM (Fig. 4(e)) and D-STM (Fig. 1(d)) well agreed that grain boundaries are more conducting than the grains in support of the dissipation measurement in Fig. 1(b)., confirming the grain boundary electron emission mechanism in doped-UNCD films.

The Hall and STM measurements clearly illustrate the effect of Pt doping/post-annealing processes on enhancing the conductivity and local electron emission sites of as-UNCD films respectively. However, the authentic reason for such an effect is not clear. The change in the microstructures of as-UNCD films due to the energetic Pt ion doping/post-annealing processes is expected to be the key. Moreover, in a local scale, the modification in the microstructure at the grain and grain boundaries of as-UNCD films is expected to have profound effect on the dissipation energy and emission properties, as observed in Fig. 1(b) and Fig. 4(b), respectively. To explore the possible cause of such a phenomena, TEM examinations were carried out in more detailed. Fig. 5(a) shows the bright field (BF) TEM microstructure of as-UNCD films with the corresponding selected area electron diffraction (SAED) pattern shown as inset. The as-UNCD films are uniformly distributed with nano-sized clusters, with large diamond aggregates occasionally observed (indicated by arrows). The corresponding SAED pattern (inset, Fig. 5(a)) contains sharp diffractions rings corresponding to the (111), (220) and (311) diamond lattices, confirming the nano-sized clusters are of diamond structure. Further, a central diffused ring observed in SAED, indicating the existence of the $\mathrm{sp}^{2}$-bonded carbon (graphitic or a-C phase) is presumably located in the grain boundaries of these films. 
The doping/post-annealing process has made significant modifications on the granular structure of as-UNCD films as shown in the BF image in Fig. 5(b). The large diamond aggregates seen in as-UNCD films were transformed to round shaped diamond grains due to the energetic Pt ion doping and subsequent post-annealing process. This indicates that the diamond aggregates marked as arrows in as-UNCD films in Fig. 5(a), are soft agglomerates. Inset in Fig. 5(b) shows that, besides the SAED pattern corresponding to the (111), (220) and (311) lattice planes of diamond, an additional diffraction ring corresponding to $\mathrm{Pt}$ (designated as $\mathrm{Pt}$ ) is present, confirming the presence of Pt nanoclusters on doped-UNCD surface. Moreover, the modifications on microstructure of as-UNCD films after the doping/post-annealing processes are illustrated by the linear diffraction patterns (LDPs, Fig. 5(c)), which are derived from the SAEDs indicated in Fig. 5(a) \& 5(b). These LDPs reveal that while as-UNCD films contain mainly diamond (spectrum (I), Fig. 5(c)), the doped-UNCD films contain an extra diffraction peak corresponding to platinum (Pt), besides the presence of diamond phase (spectrum (II), Fig. 5(c)). Moreover, there presents large proportion of graphitic phase $(\mathrm{G})$ in doped-UNCD films.

The detailed microstructure of doped-UNCD is shown by the high resolution TEM (HRTEM) micrograph. Fig. 6(a) shows the TEM structure image taken from region ' $A$ ' in Fig. 5(a), for as-UNCD films, whereas Fig. 6(b) shows the structure image of the region "B" in Fig. 5(b) for doped-UNCD films. The Fourier transformed diffractogram of the whole structure image in Fig. 6(a)), FT $_{0 a}$, shows the existence of spotted diffraction patterns arranged in a ring, the (111) D, which corresponds to randomly oriented diamond grains (D) and the diffused ring located at the center, which corresponds to a $\mathrm{sp}^{2}$-bonded carbon (a-C or nanographitic) phase. The $\mathrm{ft}_{1}$ image of the designated region ' 1 ' and the $\mathrm{ft}_{2}$ image corresponding to region ' 2 ', highlight the presence of the diamond and a-C phase, respectively. In contrast, the FT images of the whole structure image in Fig. 6(b), FTob, contain spotted diffraction patterns arranged in a ring geometry, revealing the existence of nano-sized particulates and the diffused ring, indicating the presence of the a-C (or graphitic) phase. Moreover, the $\mathrm{FT}_{0 \mathrm{~b}}$ image shows the existence of sparse diffraction spots arranged in a ring slightly larger than $(111)_{D}$ diamond ring, which corresponds to the Pt phase. Moreover, $\mathrm{FT}_{0 \mathrm{~b}}$ in Fig. 6(b), corresponding to doped UNCD films shows considerably larger intensity of central diffuse ring than $\mathrm{FT}_{0 \mathrm{a}}$, besides the diffraction spots 
related to diamond and Pt particulates, indicating a larger fraction of graphitic phase in dopedUNCD films than as-UNCD films.

Moreover, for better understanding the genuine mechanism for enhanced electron emission/conducting properties for doped-UNCD than as-UNCD, the localized bonding structures were analyzed using the carbon K-edge electron energy loss spectroscopy (EELS) to clearly distinguish between the different carbon contents such as diamond, graphite and a-C [41]. Fig. 7(a) shows the core-loss EELS spectra of both as-UNCD and doped-UNCD films containing a small hump, representing $\mathrm{sp}^{2}$-bonded carbon (285 $\mathrm{eV}, \pi^{*}$-band), besides the typical EELS signal of $\mathrm{sp}^{3}$-bonded carbon, i.e., a sharp peak at $292 \mathrm{eV}\left(\sigma^{*}\right.$-band) and a dip in the vicinity of $302 \mathrm{eV}[12,42]$. The $\pi^{*}$-band is of higher intensity for the doped-UNCD films (curve II, Fig. 7(a)), compared to as-UNCD films (curve I, Fig. 7(a)), indicating the presence of a larger proportion of $\mathrm{sp}^{2}$ bonded carbon in doped-UNCD films. In contrast, Plasmon-loss EELS spectrum of doped-UNCD shown in curve II of Fig. 7(b) contains a large diffuse peak near 27 $\mathrm{eV}$, which reveals that the $\mathrm{sp}^{2}$-bonded carbon contained in these films is graphitic in nature $[43,44]$. Particularly, for a plasmon-loss EELS spectrum corresponding to $\mathrm{sp}^{3}$-bonded carbon in a diamond material, a peak near $33 \mathrm{eV}$, represents the bulk plasma of diamond clusters with a shoulder near $23 \mathrm{eV}$, representing the surface plasma of the clusters as shown in curve I of Fig. 7(b) [44]. Based on these TEM investigations (HRTEM in Fig. 6(b) and LPDs in Fig. 6(c)), it is evident that the Pt ion doping/post-annealing processes induced the formation of conducting graphitic phases along the diamond grain boundaries in doped-UNCD films, which results in better conductivity and superior EFE properties.

\subsection{Discussion}

CITS measurements in Fig. 4(b) and the local I-V curves in S-STM (Fig. 4(e)) mode well agreed with the assumption that the grain boundaries are the conducting channels than the grains. However, these measurements can only qualitatively describe the local electron emission phenomenon and the resolution of these analysis is limited. In contrast, the energy dissipation (Fig. 1(b)) not only more clearly reveals the superior conducting/emitting channels of the grain boundaries compared to the grains with better resolution, but also analyzed these phenomena 
quantitatively. A dissipation energy difference of $\sim 2 \mathrm{eV}$ is measured between the diamond grain and the grain boundaries. The higher dissipation energy at the diamond grains than the grain boundaries is apparently due to the local Joule heating phenomena of the tunneling electrons at the diamond grains due to its less conducting nature than the grain boundaries. We found energy dissipation associated with assisted tunneling process can be a very useful technique in detecting not only the conducting or electron emitting sites on diamond surface but also can be applied for imaging heterostructured semiconducting surfaces, which can reveal many physical and chemical properties in nanometer scale. Importantly, the increase in temperature in a local scale due to local Joule heating mechanism can be useful in preparing well-ordered etched surface in nanometer or even atomic scale dominating the present lithographic technique.

It is to be noted that the conductivity of the a-C grain boundary phases of as-UNCD films is not sufficiently high, therefore limiting its conductivity and EFE properties [44, 34]. However, it has been observed that the existence of grain boundary graphitic phases, surrounding the nanosized diamond grains are the prime factors for enhancing the conductivity and EFE properties $[11,12,35-47]$. Similar conduction mechanism is proposed to explain the improved electron emission/conduction properties for doped-UNCD films. The electrons can be transported easily through the conducting nanographitic phase containing grain boundaries to the emitting surface and are then emitted to vacuum easily as the surfaces have NEA properties [4,5]. Consequently, the formations of $\mathrm{Pt}$ nanoparticles and the existence of interconnected conducting nanographitic phases at the diamond grain boundaries, are the genuine factors for the superior electron emission/conducting properties for doped-UNCD films.

This work has provided direct evidence that electron field emission from diamond surfaces originate preferentially from the diamond grain boundaries in doped-UNCD surfaces and not from the diamond grains or other topographical features. This is consistent with the model for electron field emission based on lowering of the emission threshold due to a reduction of the electron affinity of the diamond surface surrounding graphitic structures on the surface [47] and corroborates the findings of other groups [29, 30, 49, 50, 51]. As long as the grain boundaries remain relatively conducting compared to the bulk grains, electrons can travel from the contact at the reverse side of the Si substrate into the base of the film, then up to the grain boundaries and 
be emitted from the surface. Moreover, it is clear from this study that patterning diamond films into sharp cones or needles to enhance its conductivity/EFE properties is not required. Conducting diamond surfaces with large fractions of graphitic grain boundary channels or metal nanocluster incorporated diamond surfaces can provide excellent emission sources, even from flat surfaces. This finding may help to study the thermionic emission process, since the thermionic emission current depends strongly on the local surface work function, which can be affected by the presence of graphitic grain boundary phases.

\section{Conclusions}

In summary, a detailed scanning tunneling microscopy study is carried out to directly image and understand the mechanism behind the enhanced conductivity/electron field emission properties for doped-UNCD films. Direct imaging of a lower dissipation energy at the conducting grain boundaries than the grains is clearly observed by dynamic scanning tunneling microscopy. The high resolution CITS mapping and local $\mathrm{I}-\mathrm{V}$ characteristic curves revealed that the grain boundaries are the conducting/emitting sites, in support of the dissipation mapping. CITS mapping revealed that these interconnected conducting grain boundary channels throughout the doped-UNCD surface can give rise to prominent electron emission sites, resulting in higher electrical conductivity and enhanced EFE properties. The formation of nanographitic phases along the diamond grain boundaries seem to advance the conducting nature of these films

by easy transport of electrons, that is possibly the prime reason for enhanced conductivity and emission properties for doped-UNCD films.

\section{Acknowledgement}

K. Panda would like to acknowledge the financial support by the Japan Society for the Promotion of Science (JSPS) to carry out this research work. 


\section{References}

[1] W. A. de Heer, A. Chatelain, D. Ugarte, A Carbon Nanotube Field-Emission Electron Source, Science 270 (1995) 1179.

[2] K. Okano, K. Hoshina, M. Iida, S. Koizumi, T. Inuzuka, Fabrication of a diamond field emitter array, Appl. Phys. Lett. 64 (1994) 2742.

[3] T. Chang, S. Lou, H. Chen, C. Chen, C. Lee, N-H Tai, I-N Lin, Enhancing the plasma illumination behaviour of microplasma devices using microcrystalline/ultra-nanocrystalline hybrid diamond materials as cathodes, Nanoscale 5 (2013) 7467.

[4] M. W. Geis, N. N. Efremow, K. E. Krohn, J. C. Twichell, T. M. Lyszczarz, R. Kalish, J. A. Greer, M. D. Tabat, A new surface electron-emission mechanism in diamond cathodes, Nature 393 (1998) 431.

[5] H. Yamaguchi, T. Masuzawa, S. Nozue, Y. Kudo, I. Saito, J. Koe, M. Kudo, T. Yamada, Y. Takakuwa, K. Okano, Electron emission from conduction band of diamond with negative electron affinity, Phys. Rev. B 80 (2009) 165321.

[6] T. D. Corrigan, D. M. Gruen, A. R. Krauss, P. Zapol, R. P. H. Chang, The effect of nitrogen addition to $\mathrm{Ar} / \mathrm{CH}_{4}$ plasmas on the growth, morphology and field emission of ultrananocrystalline diamond, Diamond Relat. Mater. 11 (2002) 43.

[7] K. Panda, B. Sundaravel, B. K. Panigrahi, P. Magudapathy, D. N. Krishna, K. G. M. Nair, H.-C. Chen, I-N. Lin, Structural and electronic properties of nitrogen ion implanted ultra nanocrystalline diamond surfaces, J. Appl. Phys., 110 (2011) 044304.

[8] D. Zhou, A. R. Krauss, L. C. Qin, T. G. McCauley, D. M. Gruen, T. D. Corrigan, R. P. H. Chan, H. Gnaser, Synthesis and electron field emission of nanocrystalline diamond thin films grown from $\mathrm{N}_{2} / \mathrm{CH}_{4}$ microwave plasmas, J. Appl. Phys. 82 (1997) 4546.

[9] J. Li, W. Zheng, C. Gu, Z. Jin, Y. Zhao, X. Mei, Z. Mu, C. Dong, C. Sun, Field emission enhancement of amorphous carbon films by nitrogen-implantation, Carbon 42 (2004) 2309.

[10] K. J. Sankaran, Y. F. Lin, W. B. Jian, H. C. Chen, K. Panda, B. Sundaravel, C. L. Dong, N. H. Tai, I-N. Lin, Structural and Electrical Properties of Conducting Diamond Nanowires, ACS. Appl. Mater. Interfaces 5 (2013) 1294.

[11] R. Arenal, P. Bruno, D. J. Miller, M. Bleuel, J. Lal, D. M. Gruen, Diamond nanowires and the insulator-metal transition in ultrananocrystalline diamond films, Phys. Rev. B 75 (2007) 195431. 
[12] S. Bhattacharyya, O. Auciello, J. Birrell, J. A. Carlisle, L. A. Curtiss, A. N. Goyette, D. M. Gruen, A. R. Krauss, J. Schlueter, A. Sumant, P. Zapol, Synthesis and characterization of highly-conducting nitrogen-doped ultrananocrystalline diamond films, Appl. Phys. Lett. 79 (2001) 1441.

[13] Y. C. Lin, K. J. Sankaran, Y. C. Chen, C. Y. Lee, H. C. Chen, I-N. Lin, N. H. Tai, Enhancing electron field emission properties of UNCD films through nitrogen incorporation at high substrate temperature, Diamond Relat. Mater. 20 (2011) 191.

[14] W. Zhu, C. A. Randall, A. R. Badzian, R. Messier, Graphite formation in diamond film deposition, J. Vac. Sci. Technol. A 7 (1989) 2315.

[15] Y. Muto, T. Sugino, J. Shirafuji, Electrical conduction in undoped diamond films prepared by chemical vapor deposition, Appl. Phys. Lett. 59 (1991) 843.

[16] D. Hong, M. Aslam, Field emission from p - type polycrystalline diamond films, J. Vac. Sci. Technol. B 13 (1995) 427.

[17] D. Pradhan, I.N. Lin, Grain-Size-Dependent Diamond-Nondiamond Composite Films: Characterization and Field-Emission Properties, ACS Appl. Mater. Interfaces 1 (2009) 1444.

[18] J.B. Cui, J. Ristein, L. Ley, Low-threshold electron emission from diamond, Phys. Rev. B, 60 (1999) 16135.

[19] J. Robertson, Mechanisms of electron field emission from diamond, diamond-like carbon, and nanostructured carbon, J. Vac. Sci. Technol. B 17 (1999) 659.

[20] A.C. Ilie, A.C. Ferrari, T. Yagi, S.E. Rodil, J. Robertson, E. Barborini, P. Milani, Role of sp²/sp3 phase in field emission from nanostructured carbons, J. Appl. Phys. 90 (2001) 2024.

[21] P. W. May, S. Hohn, M. N. R. Ashfold, W. N. Wang, N. A. Fox, T. J. Davis, and J. W. Steeds, Field emission from chemical vapor deposited diamond and diamond-like carbon films: Investigations of surface damage and conduction mechanisms, J. Appl. Phys. 84 (1998) 1618.

[22] J. M. Garguilo, F. A. M. Kock, B. Brown, R. J. Nemanich, in Proceedings of Sixth Applied Diamond Conference/Second Frontier Carbon Technology Joint Conference (National Aeronautics and Space Administration) p. 133 (2001).

[23] J. M. Perez, C. Lin, W. Rivera, R. C. Hyer, M. Green, S. C. Sharma, D. R. Chopra, A. R. Chourasia, Scanning tunneling microscopy of the electronic structure of chemical vapor deposited diamond films, Appl. Phys. Lett. 62 (1993) 1889.

[24] F. Y. Chuang, C. Y. Sun, T. T. Chen, I. N. Lin, Local electron field emission characteristics of pulsed laser deposited diamondlike carbon films, Appl. Phys. Lett. 69 (1996) 3504. 
[25] B. L. Druz, V. I. Polyakov, A. V. Karabutov, N. M. Rossukanyi, A. I. Rukovishnicov, E. Ostan, A. Hayes, V. D. Frolov, V. I. Konov, Field electron emission from diamond-like carbon films deposited using RF inductively coupled $\mathrm{CH}_{4}$-plasma source, Diamond Relat. Mater. 7 (1998) 695.

[26] N. Pupeter, A. Gohl, T. Habermann, E. Mahner, G. Muller, H. Piel, Ph. Niedermann, W. Hanni, Field emission measurements with $\mu \mathrm{m}$ resolution on chemical - vapor - deposited polycrystalline diamond films, J. Vac. Sci. Technol. B 14 (1996) 2056.

[27] A. R. Krauss, O. Auciello, M. Q. Ding, D. M. Gruen, Y. Huang, V. V. Zhirnov, E. I. Givargizov, A. Breskin, R. Chechen, E. Shefer, V. Konov, S. Pimenov, A. Karabutov, A. Rakhimov, N. Suetin, Electron field emission for ultrananocrystalline diamond films, J. Appl. Phys. 89 (2001) 2958.

[28] A. V. Karabutov, V. D. Frolov, S. M. Pimenov, V. I. Konov, Grain boundary field electron emission from CVD diamond films, Diamond Relat. Mater. 8 (1999) 763.

[29] R. L. Harniman, Oliver J.L. Fox, W. Janssen, S. Drijkoningen, K. Haenen, P. W. May, Direct observation of electron emission from grain boundaries in CVD diamond by PeakForcecontrolled tunnelling atomic force microscopy, carbon 94 (2015) 386.

[30] V. Chatterjee, R. Harniman, P. W. May, P. K. Barhai, Direct observation of electron emission from the grain boundaries of chemical vapour deposition diamond films by tunneling atomic force microscopy, Appl. Phys. Lett. 104 (2014) 171907.

[31] H. J. Mamin, E. Ganz, D. W. Abraham, R. E. Thomson, J. Clarke, Contamination-mediated deformation of graphite by the scanning tunneling microscope, Phys. Rev., B 34 (1986) 9015(R).

[32] K. Panda, K. J. Sankaran, E. Inami, Y. Sugimoto, N. H Tai, I. N. Lin, Direct observation and mechanism for enhanced field emission sites in platinum ion implanted/post-annealed ultrananocrystalline diamond films, Appl. Phys. Lett. 105 (2014) 163109.

[33] K. J. Sankaran, J. Kurian, H. C. Chen, C. L. Dong, C. Y. Lee, N. H. Tai, I. N. Lin, Origin of a needle-like granular structure for ultrananocrystalline diamond films grown in a $\mathrm{N}_{2} / \mathrm{CH}_{4}$ plasma, J. Phys. D: Appl. Phys. 45 (2012) 365303.

[34] J. X. Hu, S. J. Ye, H. J. Liu, G. Y. Shen, H. X. Chen, H. Hu, n-type conductivity and phase transition in ultrananocrystalline diamond films by oxygen ion implantation and annealing, J. Appl. Phys. 109 (2011) 053524.

[35] J. X. Hu, S. J. Ye, H. Hu, H. X. Chen, G. Y. Shen, Phosphorus ion implantation and annealing induced n-type conductivity and microstructure evolution in ultrananocrystalline diamond films, Appl. Phys. Lett. 99 (2011) 131902. 
[36] W. Denk, D. W. Pohl, Local electrical dissipation imaged by scanning force microscopy, Appl. Phys. Lett. 59 (1991) 2171.

[37] N. Oyabu, P. Pou, Y. Sugimoto, P. Jelinek, M. Abe, S. Morita, R. Pe'rez, O. Custance, Single Atomic Contact Adhesion and Dissipation in Dynamic Force Microscopy, Phys. Rev. Lett. 96 (2006) 106101.

[38] T. D. Stowe, T. W. Kenny, D. J. Thomson, D. Rugar, Silicon dopant imaging by dissipation force microscopy, Appl. Phys. Lett. 75 (1999) 2785.

[39] T. Fukuma, K. Umeda, K. Kobayashi, H. Yamada, K. Matsushige, Experimental study on energy dissipation induced by displacement current in non-contact aomic force microscopy imaging of molecular thin films, Jpn. J. Appl. Phys. 41 (2002) 4903.

[40] Y. D. Kim, W. Choi, H. Wakimoto, S. Usami, H. Tomokage, T. ando, Direct observation of electron emission site on boron-doped polycrystalline diamond thin films using an ultra-highvacuum scanning tunneling microscope, Appl. Phys. Lett. 75 (1999) 20.

[41] A. Dato, V. Radmilovic, Z. Lee, J. Philips, M. Frenklach, Substrate-Free Gas-Phase Synthesis of Graphene Sheets, Nano. Lett. 8 (2008) 2012.

[42] D. M. Gruen, S. Liu, A. R. Krauss, J. Luo, X. Pan, Fullerenes as precursors for diamond film growth without hydrogen or oxygen additions, Appl. Phys. Lett. 64 (1994) 1502.

[43] E. J. Correa, Y. Wu, J.-G. Wen, R. Chandrasekharan, M. A. Shannon, Electrical conduction in undoped ultrananocrystalline diamond thin films and its dependence on chemical composition and crystalline structure, J. Appl. Phys. 102 (2007) 113706.

[44] P. Kovarik, E. B. D. Bourdon, R. H. Prince, Electron-energy-loss characterization of laserdeposited a-C, a-C:H, and diamond films, Phys. Rev. B 48 (1993) 12123.

[45] K. Panda, B. Sundaravel, B. K. Panigrahi, P. C. Huang, W. C. Shih, H. C. Chen, I-N. Lin, Direct observation and mechanism of increased emission sites in Fe-coated microcrystalline diamond films, J. Appl. Phys. 111 (2012) 124309.

[46] K. Panda, B. Sundaravel, B. K. Panigrahi, H.-C. Chen, P.-C. Huang, W.-C. Shih, S.-C. Lo, L. J. Lin, C.-Y. Lee, I-N. Lin, The induction of nanographitic phase on Fe coated diamond films for the enhancement in electron field emission properties, J. Appl. Phys. 113 (2013) 094305.

[47] K. Panda, K. J. Sankaran, B. K. Panigrahi, N.-H. Tai, I-N. Lin, Direct Observation and Mechanism for Enhanced Electron Emission in Hydrogen Plasma-Treated Diamond Nanowire Films, ACS Appl. Mater. Interfaces 6 (2014) 8531.

[48] J.B. Cui, J. Ristein, M. Stammler, K. Janischowsky, G. Kleber, L. Ley, Hydrogen termination and electron emission from CVD diamond surfaces: a combined secondary 
electron emission, photoelectron emission microscopy, photoelectron yield, and field emission study, Diamond Relat. Mater. 9 (2000) 1143.

[49] V. Yanef, M. Rommel, M. Lemberger, S. Petersen, B. Amon, T. Erbacher, A.J. Bauer, H. Ryssel, A. Paskaleva, W. Weinreich, Tunneling atomic-force microscopy as a highly sensitive mapping tool for the characterization of film morphology in thin high-kk dielectrics, Appl. Phys. Lett. 92 (2008) 252910.

[50] V. D. Frolov, S. M. Pimenov, V. I. Konov, V. I. Polyakov, A. I. Rukovishnikov, N. M. Rossukanyl, J. A. Carlisle, D. M. Gruen, Electronic properties of low - field - emitting ultrananocrystalline diamond films, Surf. Interface Anal. 36 (2004) 449.

[51] P. Philipp, L. Bischoff, U. Treske, B. Schmidt, J. Fiedler, R. Hubner, F. Klein, A. Koitzsch, T. Muhl, The origin of conductivity in ion-irradiated diamond-like carbon-Phase transformation and atomic ordering, Carbon 80 (2014) 677. 
Table 1. Comparison of the electrical conductivity and turn-on field for different UNCD films.

\section{Table 1}

\begin{tabular}{cccc}
\hline \hline Materials & $\begin{array}{c}\text { Electrical } \\
\text { conductivity } \\
(\Omega \mathrm{cm})^{-1}\end{array}$ & $\begin{array}{c}\text { Turn-on field } \\
(\mathrm{V} / \mu \mathrm{m})\end{array}$ & References \\
\hline \hline $\begin{array}{c}\mathrm{N}_{2} \text { doped UNCD } \\
\mathrm{O}_{2} \text { ion doped } \\
\text { UNCD/annealing }\end{array}$ & 200 & 6.13 & 33 \\
$\begin{array}{c}\mathrm{P} \text { ion doped } \\
\text { UNCD/annealing }\end{array}$ & 33.3 & - & 34 \\
as-UNCD & 0.09 & - & 35 \\
doped-UNCD & - & 22.2 & Present study \\
& 95 & 4.17 & Present study
\end{tabular}



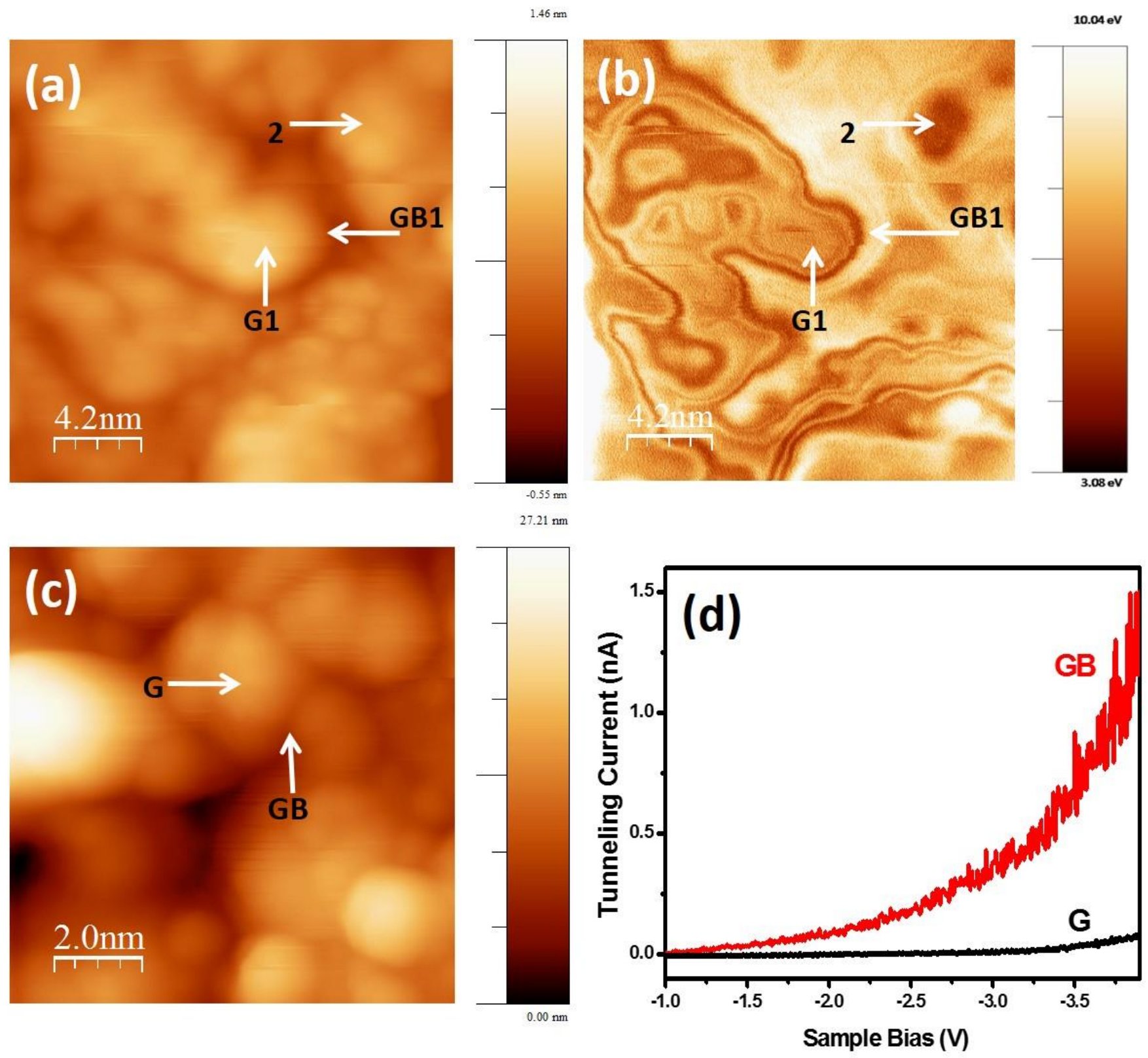

FIG. 1. (a) Dynamic STM (D-STM) image and (b) the corresponding dissipation mapping. (c) HRSTM image of doped-UNCD films. (d) The local I-V curves at the grain (G) and grain boundary (GB) corresponding to the locations designated in "c". 
(a)

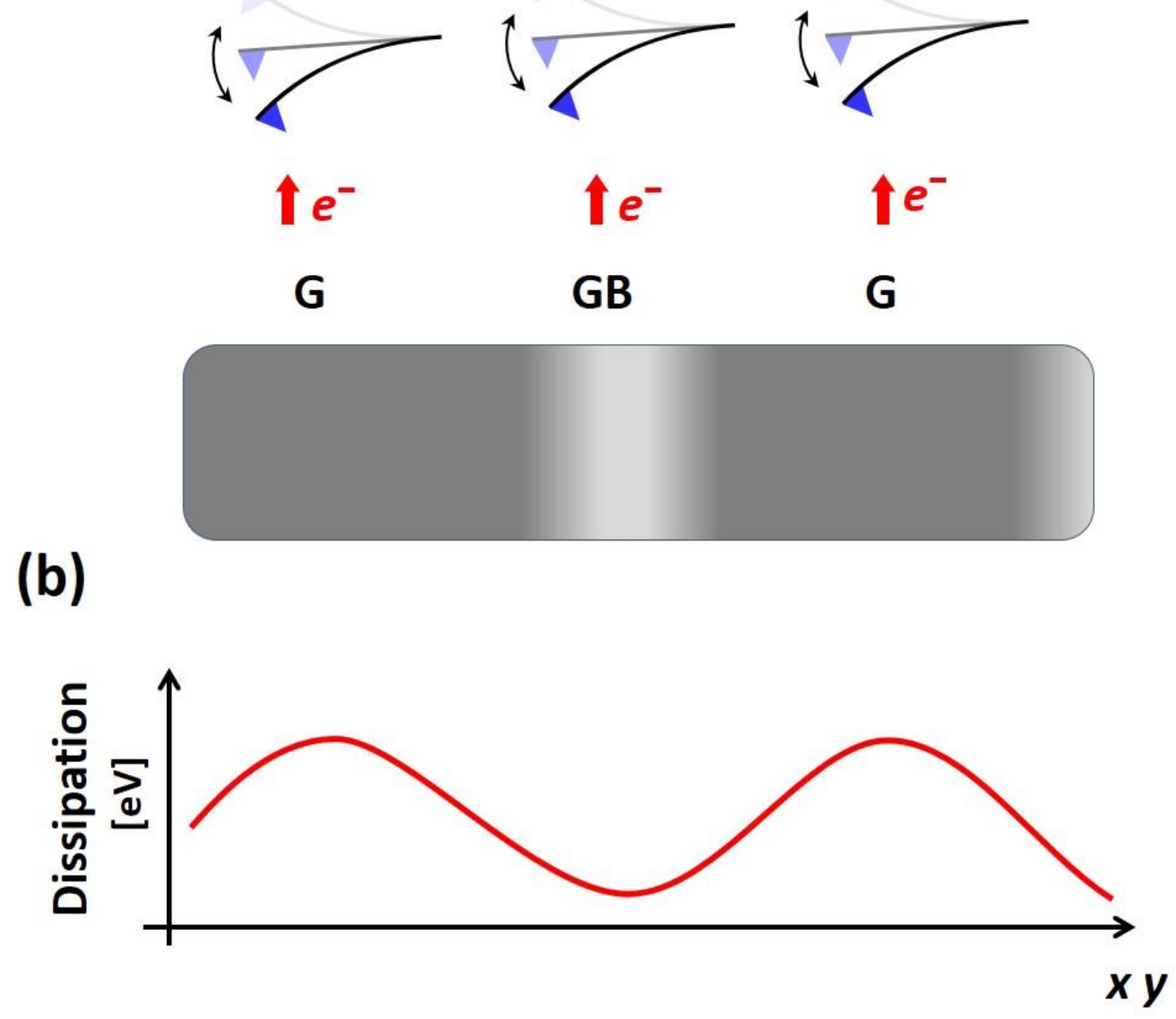

FIG. 2. Schematic representation of (a) STM cantilever scanning on the diamond grain and grain boundary with (b) showing the dissipation energy at the respective positions. 

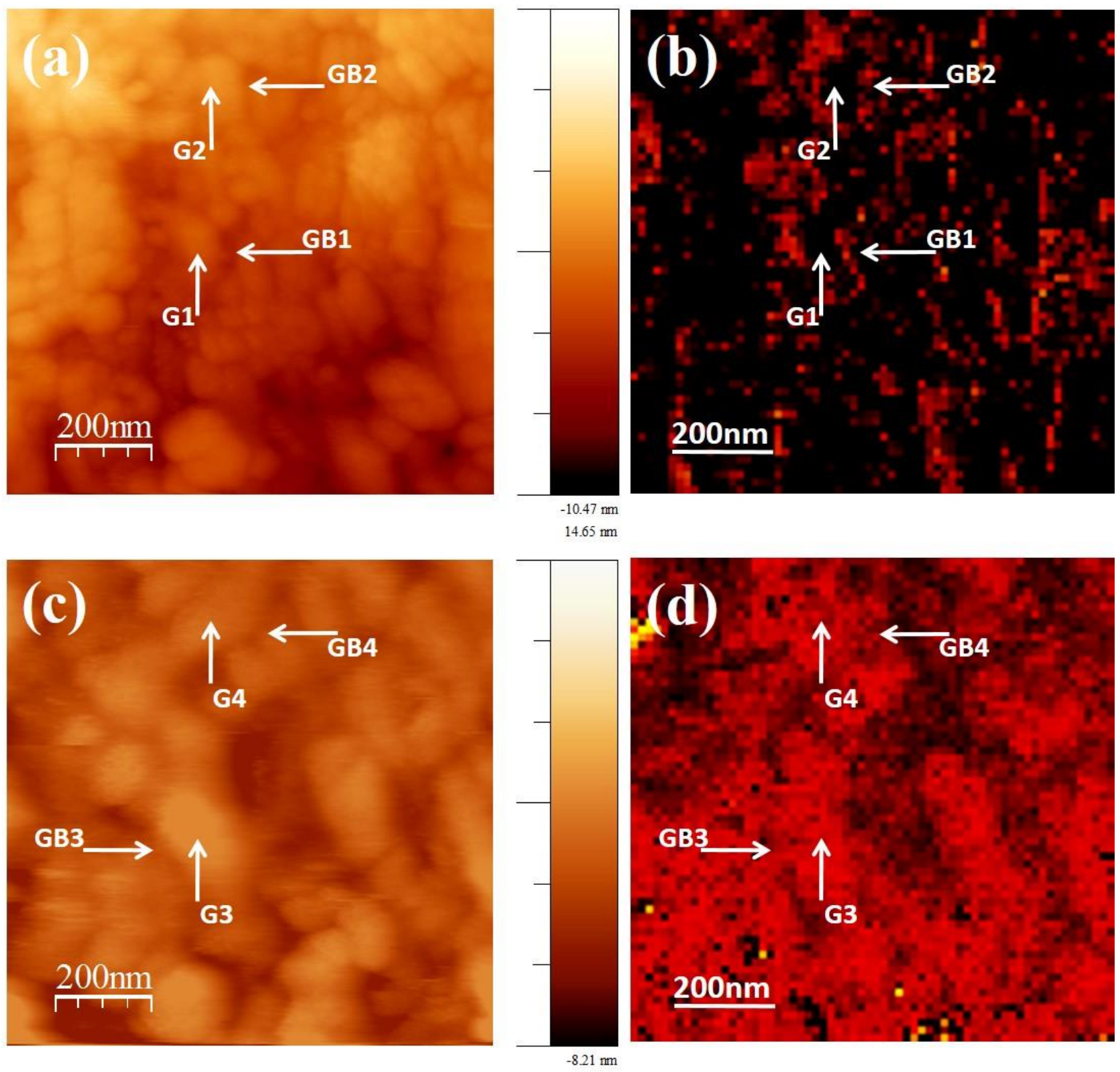

FIG. 3. (a, c) The STM image with their corresponding CITS image in (b, d) for $(a, b)$ as-UNCD and (c, d) doped-UNCD films, respectively. The CITS images were acquired at a negative sample bias of $3.0 \mathrm{~V}$. 

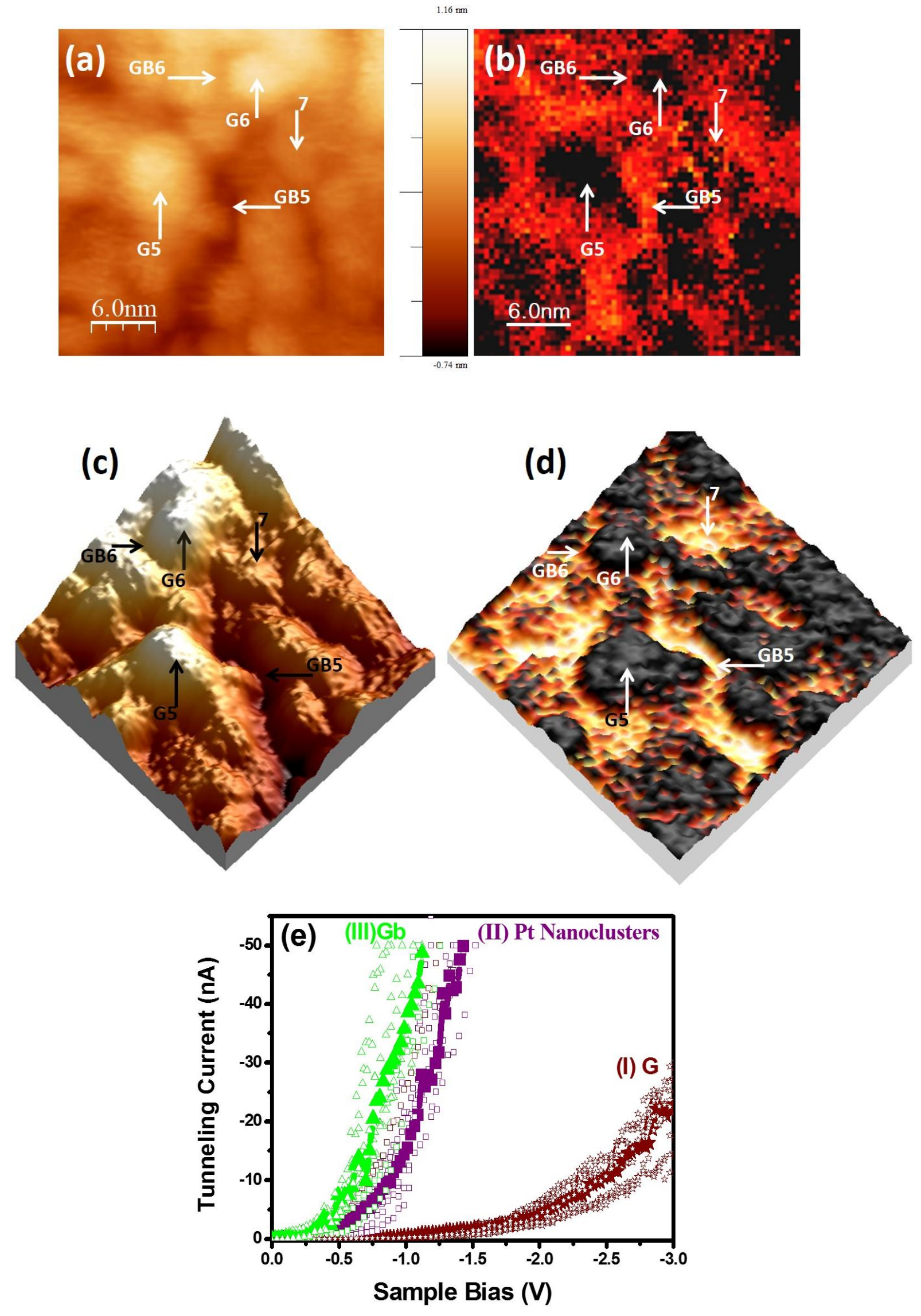

FIG. 4. (a) HRSTM image with the corresponding CITS shown in (b) for doped-UNCD films. (c, d) 3-D surface representation of (c) HRSTM and (d) CITS, respectively. (e) The local I-V characteristics curves at the (I) diamond grain (marked as G5, G6 in (a)), (II) Pt nanoparticles (marked as 7 in (a)), and (III) diamond grain boundaries (marked as GB5 and GB6 in (a)). Each spectrum shown as dark thick line in (e) is the average of 10 scanned spectra at the designated points. 

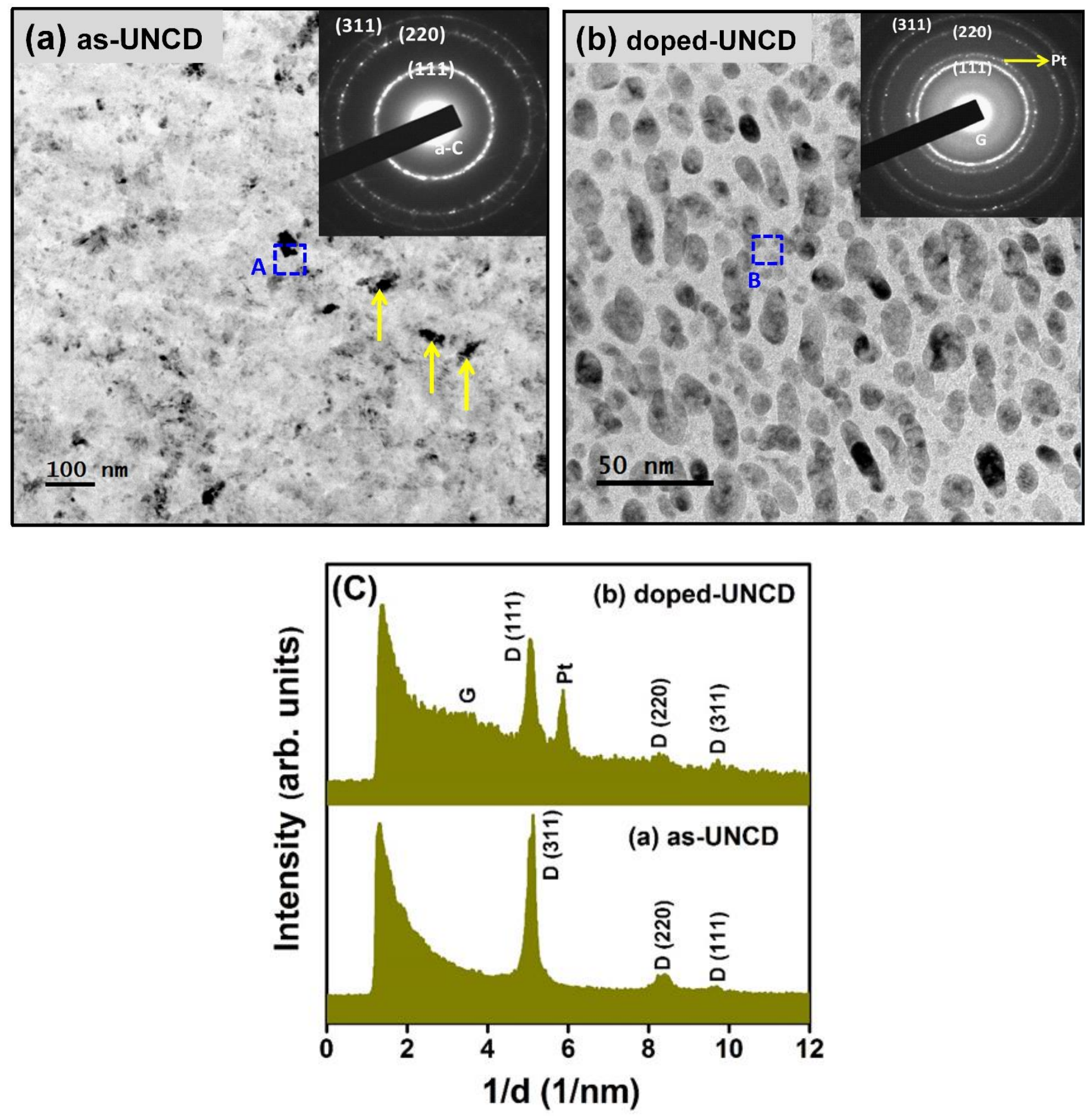

Fig. 5. Bright field TEM images of (a) as-UNCD and (b) doped-UNCD films with their corresponding SAED patterns shown as insets. (c) The linear diffraction patterns (LDP) derived from the SAED patterns shown in "a" and "b", correspond to the (I) as-UNCD and (II) dopedUNCD films, respectively. 

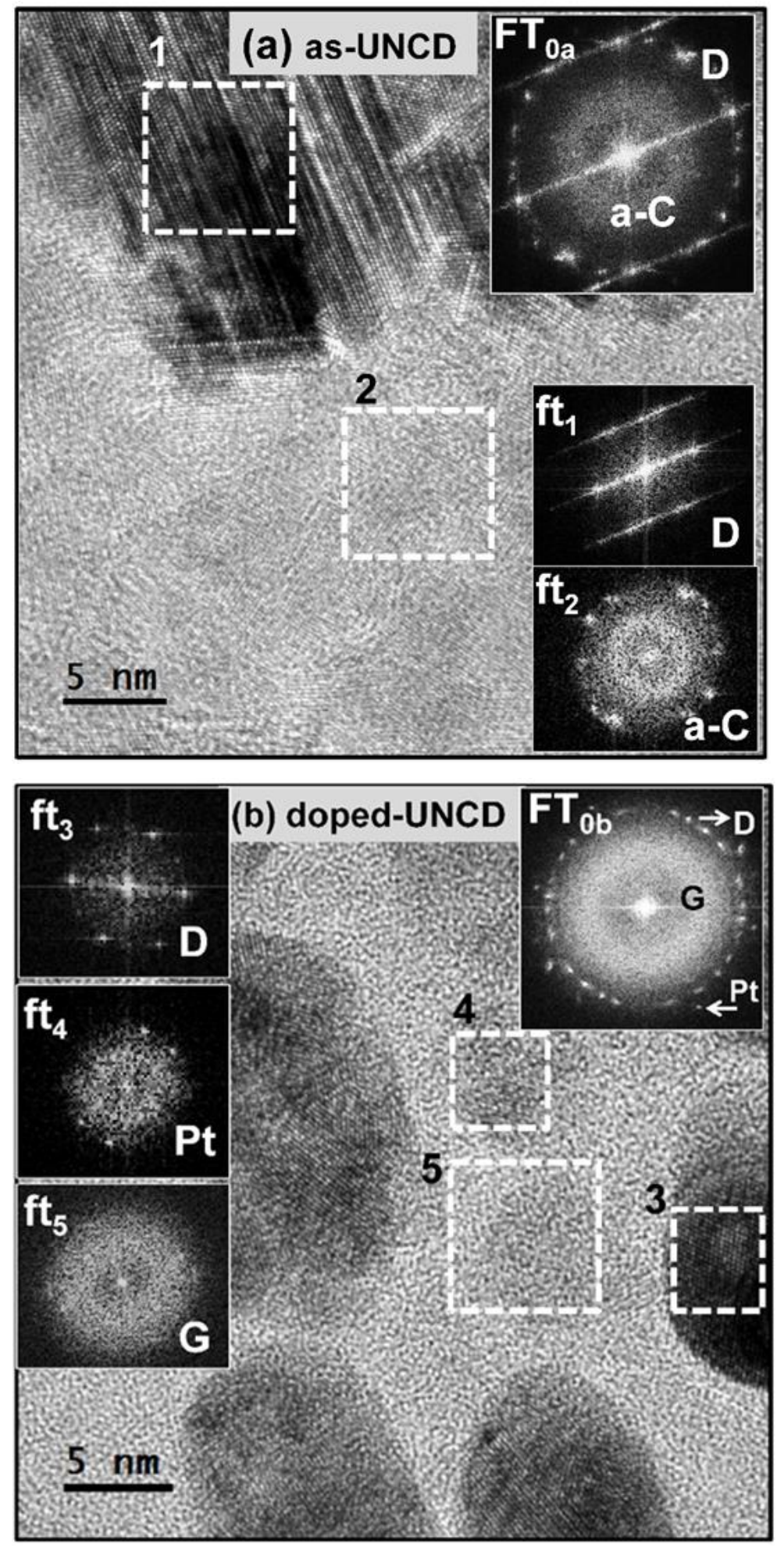

Fig. 6. HRTEM image of (a) as-UNCD films corresponding to the region 'A' of Fig. 5(a) and of (b) doped-UNCD films corresponding to the region 'B' in Fig. 5(b). The insets, $\left(F_{0 a}\right)$ and $\left(F_{0 b}\right)$, show the Fourier-transformed images corresponding to the whole structure image in "a" and "b", respectively, whereas $\mathrm{ft}_{1}$ to $\mathrm{ft}_{5}$ images show the FT images corresponding to regions ' 1 ' to ' 5 ', respectively. 

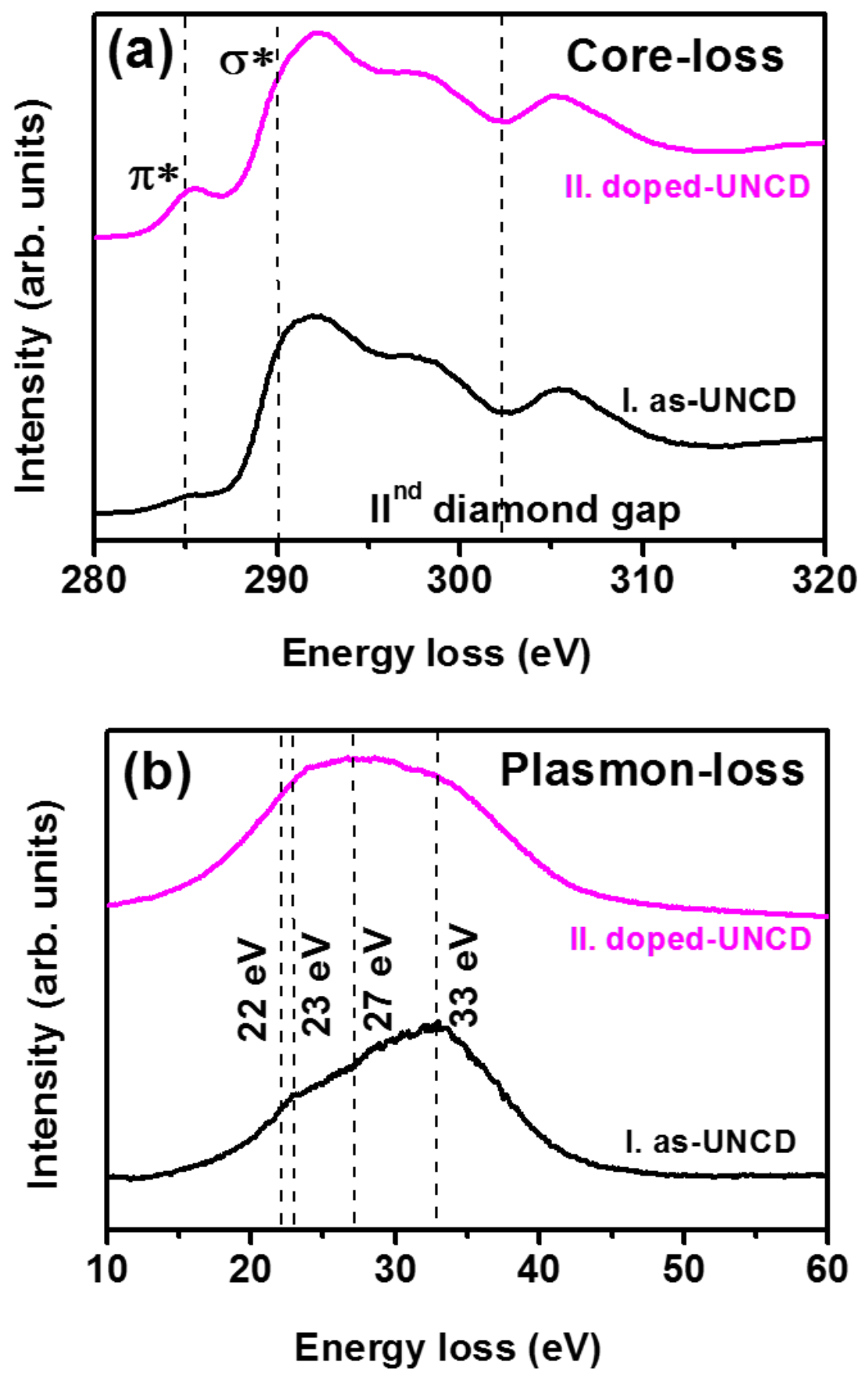

Fig. 7. (a) Carbon edge core-loss and (b) plasmon-loss EELS spectra of (I). as-UNCD and (II). doped-UNCD films. 